\title{
Phonon-induced dephasing in single-wall carbon nanotubes
}

\author{
D. T. Nguyen ${ }^{1}$, C. Voisin ${ }^{1}$, Ph. Roussignol ${ }^{1}$, C. Roquelet ${ }^{2}$, J. S. Lauret ${ }^{2}$, and G. Cassabois ${ }^{1,3,4, *}$ \\ ${ }^{1}$ Laboratoire Pierre Aigrain, Ecole Normale Supérieure, UPMC, Université Paris Diderot, \\ CNRS UMR8551, 24 rue Lhomond 75231 Paris Cedex 5, France \\ ${ }^{2}$ Laboratoire de Photonique Quantique et Moléculaire de l'Ecole Normale Supérieure de Cachan, \\ CNRS UMR8537, 61 avenue du Président Wilson, 94235 Cachan Cedex, France \\ ${ }^{3}$ Université Montpellier 2, Laboratoire Charles Coulomb UMR5221, F-34095, Montpellier, France \\ ${ }^{4}$ CNRS, Laboratoire Charles Coulomb UMR5221, F-34095, Montpellier, France
}

(Dated: September 8, 2011)

\begin{abstract}
We report original nonlinear spectral hole-burning experiments in ensembles of single-wall carbon nanotubes. We have measured the temperature dependence of the excitonic homogeneous linewidth from $5 \mathrm{~K}$ up to room temperature. We show that the phonon-induced broadening in the weak coupling regime predominantly consists in pure dephasing. We present theoretical calculations of the exciton scattering rate due to phonon absorption within a one-dimensional framework for both excitons and phonons. We obtain a fair agreement with our experimental data and we discuss the specific properties of the exciton-phonon coupling in single-wall carbon nanotubes.
\end{abstract}

PACS numbers: 78.67.Ch, 71.35.-y, 78.47.nd

Single wall carbon nanotubes (SWNTs) are pure carbon-based nanostructures characterized by a nanometric radial quantum confinement and a translation invariance along the tube axis [1]. The corresponding onedimensionality of carriers results in strong Coulomb correlations and in particular to a non-perturbative binding of an electron-hole pair into an exciton [2-5]. Compared to epitaxially grown semiconductor quantum wires with much lower excitonic binding energies [6], SWNTs appear as a model system with quasi-1D excitonic properties. The originality of SWNTs is even more pronounced when considering the exciton-phonon coupling. In SWNTs the vibrational excitations are also quasi-1D whereas quantum wires, and more generally epitaxially grown nanostructures, are embedded in a semiconductor crystalline matrix and thus interact with $3 \mathrm{D}$ phonons [7-10]. The exciton-phonon coupling is at the heart of the thermallyassisted carrier relaxation, and its understanding is of great interest both from a fundamental point of view and for the development of carbon-based optoelectronic devices operating at room-temperature.

The existence of phonon sidebands was identified as a specific feature of the strong exciton-phonon coupling in semiconducting SWNTs $[11,12]$. This interaction reflects in a dynamical structural distortion which contributes in binding energies of the order of hundreds of meV [11]. The variety of the exciton-phonon interaction in SWNTs is further illustrated by the evidence of a broad zerophonon line at low temperature, which strongly contrasts with the phenomenology observed in zero-dimensional nanostructures [13]. In fact, the study of the temperature dependence of the excitonic homogeneous linewidth provides a direct approach for accessing the intrinsic characteristics of the exciton-phonon coupling in SWNTs. However, optical measurements in ensembles of SWNTs always suffer from inhomogeneous broadening due to chiral variety or environment fluctuations. Such an issue can be experimentally addressed by performing measure- ments either at the scale of a single SWNT [13-19], or in SWNTs ensembles by means of nonlinear techniques such as four-wave mixing [20]. There is nevertheless a large dispersion in the quantitative analysis of the different temperature-dependent experiments $[17,18,20]$. Moreover, no theoretical calculation was reported so that there is still a lack for a quantitative interpretation of the phonon-induced broadening and its diameter dependence [18].

In this paper, we report original nonlinear spectral hole-burning experiments in SWNTs ensembles that allow us to study the temperature dependence of the excitonic homogeneous linewidth from $5 \mathrm{~K}$ up to room temperature. We compare our measurements to the specific contribution due to phonon-assisted population relaxation and we find that pure dephasing dominates the phonon-induced broadening in SWNTs. In order to reach a quantitative interpretation of our data, we have calculated the exciton scattering rate by phonon absorption within a 1D framework for both excitons and phonons. From the fair agreement with our experimental values, we discuss the peculiar 1D properties of the exciton-phonon interaction in SWNTs.

Our sample consists of SWNTs synthetized by laser ablation of a doped graphite target and purified by ultrasonic and chemical treatment $[21,22]$. The linear absorption spectrum is composed of inhomogeneously broadened lines centered at 0.75 and $1.3 \mathrm{eV}$ corresponding to the $\mathrm{S}_{11}$ and $\mathrm{S}_{22}$ excitonic transitions in semiconducting SWNTs, respectively [23]. Our work is focused on the fundamental $\mathrm{S}_{11}$ transition (Fig.1, inset). In order to suppress the influence of external non-radiative relaxation processes as much as possible, the SWNTs are first isolated by the micelle-wrapping technique which allows the observation of a bright photoluminescence signal $[24,25]$. The individual SWNTs are further embedded in a gelatin matrix following the procedure described in Ref.[26], in order to perform temperature-dependent measurements. 
Homogeneous linewidth measurements are perfomed by means of nonlinear spectral hole-burning experiments. Our experimental scheme is based on differential transmission experiments in a spectrally resolved cw pumpprobe setup [27]. The pump laser is a semiconductor distributed feed-back laser seeding an erbium-doped fiber amplifier. Its output is coupled to a monomode fiber providing up to $1 \mathrm{~W}$ at $0.8 \mathrm{eV}(1.55 \mu \mathrm{m})$ within a spectral width $\delta \gamma<100 \mu \mathrm{eV}$, and it is focused on a spot of $6 \mu \mathrm{m}$-diameter. The probe laser is an external-cavity semiconductor laser tunable from $1.52 \mu \mathrm{m}$ to $1.6 \mu \mathrm{m}$. The pump-induced variation $\Delta T$ of the probe transmission $T$ is detected by a lock-in amplifier at the frequency $f_{1}+f_{2}$, where $f_{1}$ and $f_{2}$ are the modulation frequencies of the pump and probe laser intensity, respectively. The orthogonally-propagating laser beams are both incident at $45^{\circ}$ on the sample mounted on the cold finger of a continuous-flow helium cryostat for measurements down to $5 \mathrm{~K}$.

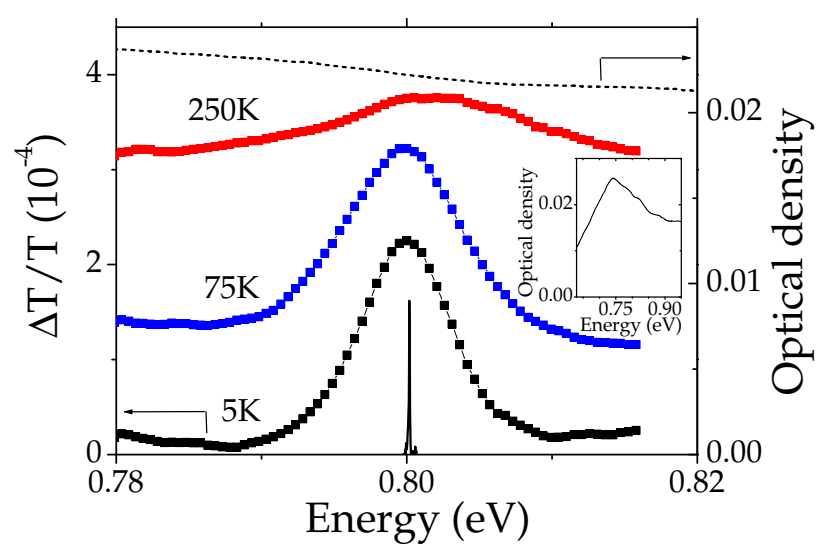

FIG. 1. (Left axis) Differential transmission $\Delta T / T$ (squares) of the probe laser as a function of energy with a pump laser of $4 \mathrm{~kW} \cdot \mathrm{cm}^{-2}$ at $0.8 \mathrm{eV}$, for three different temperatures: 5,75 and $250 \mathrm{~K}$ (the two upper curves are shifted for a better visibility). System response function (solid line) of the nonlinear spectral hole-burning setup. (Right axis) Optical density spectrum (dotted line) obtained by linear absorption spectroscopy of the carbon nanotubes gelatine sample. Inset: optical density spectrum on a larger scale.

The narrow pump laser centered at $0.8 \mathrm{eV}$ partially saturates the absorption of the SWNTs that are in resonance (Fig.1). The corresponding sub-ensemble of SWNTs reduces the overall absorption of the sample in a spectral window determined by the homogeneous linewidth $\Gamma$. The photo-induced bleaching of the SWNTs sample is detected by recording the change of transmission of the tunable probe laser. A typical spectrum of the pumpinduced differential transmission $\Delta T / T$ of the probe laser is displayed in Fig.1, for a pump power of $4 \mathrm{~kW} . \mathrm{cm}^{-2}$.
At low temperature, the nonlinear signal exhibits a pronounced bleaching with an amplitude of $2.10^{-4}$ and a full width at half maximum (FWHM) of $7 \mathrm{meV}$, which is much smaller than the inhomogeneous broadening of the linear absorption spectrum (dashed line, Fig.1), and much larger than the system response function width (solid line, Fig.1).

In the case of excitonic transitions, the saturation of absorption occurs for bosonic excitations so that it can not be due to a redistribution of an electronic population among the ground and excited states as in atomic systems, but to a change of the oscillator strength $f$ and homogeneous linewidth $\Gamma$ of the excitonic transition, as described in Ref.[28]. The analysis of power-dependent measurements performed on the same sample [29] indicated that the major nonlinearity in SWNTs arises from the pump-induced change $\Delta \Gamma$ of homogeneous linewidth and that the oscillator strength renormalization $\Delta f$ is marginal. This implies that the width of the spectral hole is given by $\Gamma$, instead of the standard $2 \Gamma$ value as in the case of absorption saturation in atomic systems [30].

The temperature dependence of the excitonic homogeneous linewidth is obtained by measuring the FWHM of the spectral hole-burning signal as a function of temperature. When raising the temperature, the differential transmission $\Delta T / T$ spectra become broader (Fig.1), as expected from the phonon-induced broadening of the excitonic transition. Above $150 \mathrm{~K}$, the limited spectral range of our probe laser does not allow us to accurately determine the intensity of the background signal at large pump-probe detuning. This explains the reduction of the relative precision of our measurements of $\Gamma$ close to room temperature (Fig.2, inset).

The thermal broadening is fairly reproduced by the expression [17, 18, 20]:

$$
\Gamma(T)=\Gamma_{0}+a T+b \exp \left(-\frac{E_{0}}{k_{B} T}\right)
$$

where $\Gamma_{0}$ is the residual homogeneous linewidth in the limit of zero temperature (in the range of several meVs $[13,16-18])$, aT the broadening due to low-energy acoustic phonons, and $b \exp \left(-\frac{E_{0}}{k_{B} T}\right)$ the contribution of highenergy phonons, such as radial breathing modes, that dominates above $75 \mathrm{~K}$. While Ma et al. fit their data with $a=0, b=254 \mathrm{meV}$ and $E_{0}=105 \mathrm{meV}$ [20], Yoshikawa et al. take on the contrary $b=0$ with $a$ ranging from 20 to 35 $\mu \mathrm{eV} . \mathrm{K}^{-1}[18]$. In our case, the fit displayed as solid red line in Fig. 2 is obtained by taking $\Gamma_{0}=6.9 \pm 0.2 \mathrm{meV}$, and $a=32 \pm 3 \mu \mathrm{eV} \cdot \mathrm{K}^{-1}$. Given the reduced precision of our measurements above 150K (Fig.2, inset), the estimation of $E_{0}$ and $b$ is more problematic. Even if the coupling to high-energy phonons is not at the heart of this paper, we have tried to obtain some estimates. Our data can be fitted by varying $E_{0}$ from 20 and $75 \mathrm{meV}$, while $b$ changes from 10 to $250 \mathrm{meV}$, respectively. By fixing $E_{0}$ to the energy $E_{R B M}$ of the radial breathing mode at the zone center, we get $b=11 \pm 4 \mathrm{meV}$ with $E_{R B M} \sim 25$ 
meV in our SWNTs for which the diameter is about 1.2 $\mathrm{nm}$ [31]. In the following, we will specifically discuss the linear temperature dependence below $75 \mathrm{~K}$ corresponding to low-energy acoustic phonons.

An interesting question is the contribution of puredephasing in addition to population relaxation in the phonon-assisted broadening in SWNTs. The pure dephasing is usually characterized by the $T_{2}^{\star}$ time given by $1 / T_{2}=1 / 2 T_{1}+1 / T_{2}^{\star}$ where $T_{1}$ and $T_{2}$ are the population and coherence relaxation times, respectively. Timeresolved photoluminescence showed that the intrinsic recombination rate increases linearly with temperature up to $300 \mathrm{~K}[26]$. Such a process provides a linear contribution $\tilde{a} T$ to $\Gamma(T)$ shown by the red dashed line in Fig.2, with $\tilde{a} \sim 0.04 \mu \mathrm{eV} . \mathrm{K}^{-1}[26]$. Since $\tilde{a} \ll a$, we conclude that phonon-assisted processes predominantly lead to pure dephasing in SWNTs.

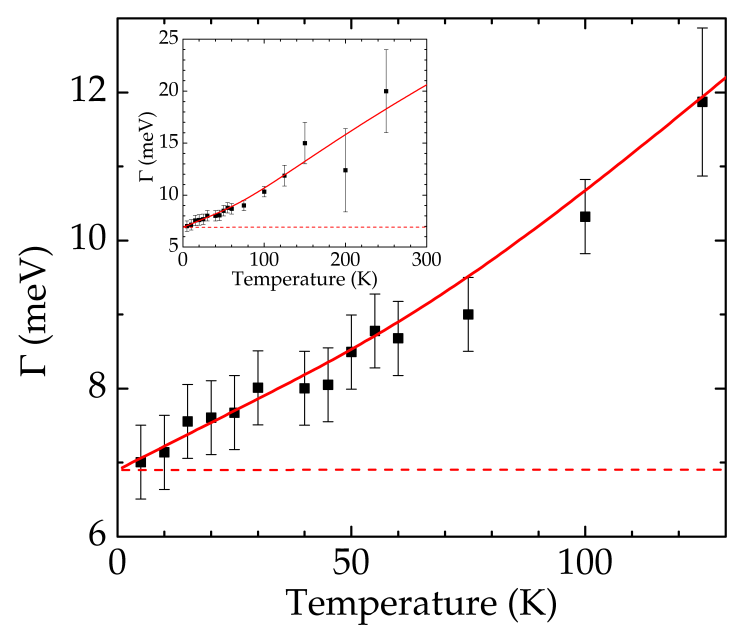

FIG. 2. Temperature dependence of the excitonic homogeneous linewidth: data (squares), theoretical fits (red solid line). The dashed red line indicates $\Gamma_{0}+\tilde{a} T$ where $\tilde{a} T$ is the temperature dependence of the population relaxation rate, from Ref. [26]. Inset: same display on a larger temperature range.

Although phonon-induced broadening was experimentally investigated by means of various techniques for SWNTs of different diameters [13, 16-18, 20], there is so far no theoretical calculation of the exciton-phonon coupling efficiency in these $1 \mathrm{D}$ systems. In order to provide a quantitative interpretation of our measurements, we have calculated the temperature-dependence of the excitonic homogeneous linewidth due to the interaction with acoustic phonons. For that purpose we have transposed in $1 \mathrm{D}$ the theoretical treatment developped for $3 \mathrm{D}$ excitons coupled to 3D phonons [9]. More specifically, we have considered the deformation potential interaction of 1D excitons with 1D phonons that consist of low-energy acoustic phonon modes such as twisting ( $\mathrm{T}$ ) and stretching $(\mathrm{S})$ modes [33]. The assumption of $1 \mathrm{D}$ phonon is supported by the acoustical impedance mismatch of one order of magnitude between the carbon nanotubes and the gelatin. In the long-wavelength limit, the coupling constants $u_{q}^{e, h}$ of an electron (e) or a hole (h) to a phonon of wave vector $q[9]$ read in $1 \mathrm{D}$ for $\mathrm{T}$ and $\mathrm{S}$ modes:

$$
u_{q}^{e, h}=\sqrt{\frac{\hbar q}{2 \rho v L}} D_{e, h} \sqrt{F_{\phi_{c}}}
$$

where $v$ is the longitudinal sound velocity [33], $\rho$ the linear mass density, $L$ the nanotube length, $D_{e, h}$ the deformation potential constant, and $F_{\phi_{c}}$ the chiralitydependent coupling factor of the $\mathrm{T}$ and $\mathrm{S}$ modes defined in Ref. [33].

The homogeneous broadening due to the excitonphonon coupling is then given by the scattering rate of excitons at $k=0$ due to phonon absorption, calculated by the Fermi golden rule [9]. This scattering rate is proportional to the Bose-Einstein occupation factor $n(T)$ of the involved phonons. In the case of low-energy phonons, $n(T)$ becomes linear for temperatures larger than $T_{a}$ where $k_{B} T_{a}$ is the characteristic energy of the acoustic phonons involved in the scattering processes. In the present case, the linear term aT in Eq.1 thus accounts for the coupling to the $\mathrm{T}$ and $\mathrm{S}$ phonon modes. On the contrary, for high-energy phonons, $n(T)$ is approximated by a classical Boltzmann factor as long as the temperature is much smaller than $E_{0} / k_{B}$ where $E_{0}$ is the characteristic phonon energy, given in the present case by $E_{R B M}$ equal to $25 \mathrm{meV}$ in our $1.2 \mathrm{~nm}$ diameter SWNTs [31].

We focus on the linear term $a T$ corresponding to the pure-dephasing due to $\mathrm{S}$ and $\mathrm{T}$ phonons and we provide below a quantitative interpretation of our estimation of $a=32 \pm 3 \mu \mathrm{eV} \cdot \mathrm{K}^{-1}$. Pure dephasing processes only consist in intraband scattering within the fundamental excitonic band. Energy and momentum conservation laws impose that the mean acoustic phonon energy is given by $2 M v^{2}$ where $M=m_{e}+m_{h}$ is the sum of the electron and hole effective masses. With $v=20 \mathrm{~km} . \mathrm{s}^{-1}[32,33]$ and $M=0.2 m_{0}$ where $m_{0}$ is the free electron mass [1], we obtain a value of $0.9 \mathrm{meV}$. This justifies the linear approximation of the occupation factor for $T \gtrsim 10 \mathrm{~K}$. As far as the chirality-dependent factor $F_{\phi_{c}}$ is concerned, it is difficult to get an estimate since we perform ensemble measurements where the pump laser saturates SWNTs of different chiralities within the excitonic homogeneous linewidth $\Gamma$. Since $F_{\phi_{c}}$ varies from 1.45 to 1.95 as a function of the chiral angle $\phi_{c}$ [33], we approximate it by its mean value $\bar{F} \sim 1.7$ in our calculations. Finally, we obtain for the average broadening efficiency $a$ the following expression:

$$
a=\frac{k_{B} \bar{F}}{\rho \hbar v^{3}}\left|D_{e} \frac{1}{1+\left(\frac{2 r_{B} m_{e} v}{\hbar}\right)^{2}}-D_{h} \frac{1}{1+\left(\frac{2 r_{B} m_{h} v}{\hbar}\right)^{2}}\right|^{2}
$$

where $r_{B}$ is the exciton Bohr radius. The ratios $\frac{2 r_{B} m_{e, h} v}{\hbar}$ express cutoffs in reciprocal space for phonons 
of wavevectors smaller than the Bohr radius. With $r_{B}$ in the range of few nms [3], they are of the order of 0.1 and are neglected in Eq.3. As a matter of fact, the average broadening efficiency takes the simple expression:

$$
a \simeq \frac{k_{B} \bar{F}}{\rho_{0} d \hbar v^{3}}\left|D_{e}-D_{h}\right|^{2}
$$

where we have rewritten the linear mass density as $\rho_{0} d$ (with $\rho_{0}$ the value for $1 \mathrm{~nm}$ SWNTs, and $d$ the SWNT diameter in $\mathrm{nm}[33])$. We would like to highlight that the exciton mass $M$ does not appear in Eq.4 whereas $a$ scales like $M^{2}$ in 3D [9] and $M$ in 2D [10]. This effect arises from the modification of the density of states with the exciton dimension $p$ which results in a $M^{p-1}$ dependence. The striking absence of the exciton mass $M$ in Eq.4 is thus specific to $1 \mathrm{D}$ systems and it leads to a situation where the acoustic phonon dephasing efficiency only depends on characteristic constants of the phonon modes.

The quantitative interpretation of our experimental data thus brings a direct estimation of the deformation potential constant $D_{e}-D_{h}$ for low-energy acoustic phonon modes, which is poorly documented in the literature contrarily to the corresponding ones for radial breathing modes [34-36]. Note that due to the mirror symmetry of the conduction and valence bands in SWNTs, $m_{e} \simeq m_{h}$, and one expects $D_{h} \simeq-D_{e}$ so that $D_{e}-D_{h}$ does not vanish, as expected for the deformation potential interaction where the electron and hole couplings are additive instead of substractive as for the Fröhlich interaction with optical phonons [9]. With $\rho_{0}=1.9 \times 10^{-15} \mathrm{~kg} . \mathrm{m}^{-1}$ [33], $v=20 \mathrm{~km} . \mathrm{s}^{-1}[32,33]$, the fitted value of $a=32 \pm 3$ $\mu \mathrm{eV} . \mathrm{K}^{-1}$ leads to $D_{e}-D_{h}$ of $4 \mathrm{eV}$, which is smaller than the theoretical value of $8 \mathrm{eV}$ in Ref. [33] (labelled $D_{A}$ in this reference), whereas Galland et al. deduce a larger one of $14 \mathrm{eV}$ from their experiments [13].

Eventually, we discuss the $1 / \mathrm{d}$ dependence of the $a$ coefficient which was experimentally reported by Yoshikawa et al. [18]. Their interpretation is based on the analogy with epitaxial semiconductor quantum wells or wires where the $a$ coefficient scales like the inverse of the nanostructure width $W[7,8]$. We show here that the underlying physics is however radically different in the two cases. In quantum wells or wires, the $1 / \mathrm{W}$ dependence arises from the coupling of $2 \mathrm{D}$ and $1 \mathrm{D}$ excitons to phonons of higher dimensionality (3D) since these epitaxial nanostructures are embedded in a crystalline matrix. Consequently, the characteristic component of the phonon wavevector along the confinement directions is determined by the inverse of the nanostructure width $W$ because of the wavefunction matching condition that ensures a maximum matrix element for the exciton-phonon interaction [10]. In our theoretical model where 1D excitons interact with $1 \mathrm{D}$ phonons, the $1 / \mathrm{d}$ dependence can not have the same origin. As a matter of fact, the observed $1 / \mathrm{d}$ dependence of the $a$ coefficient [18] comes from the linear mass density variation as a function of the nanotube diameter (Eq.4), which is is radically different from $1 / \mathrm{W}$ cut-off of the phonon wavevector in epitaxial quantum wells and wires.

In summary, we have studied the temperature dependence of the excitonic homogeneous linewidth by nonlinear spectral hole-burning experiments in ensembles of SWNTs. We find that the phonon-induced broadening predominantly consists in pure dephasing. We have developped theoretical calculations of the exciton scattering rate by phonon absorption within a one-dimensional framework for both excitons and phonons. We provide a quantitative interpretation of our experiments. Moreover, we show that the $1 / \mathrm{d}$ dependence of the acousticphonon dephasing efficiency is specific to the excitonphonon interaction in the 1D systems formed by SWNTs and that it originates from the linear variation of the nanotube linear mass density with diameter.

We acknowledge fruitful discussions with T. Michel, M. Paillet, and J. L. Sauvajol. This work was financially supported by the CNano IdF contract TAIGA and the ANR agency-contract BONAFO.

*e-mail: Guillaume.Cassabois@univ-montp2.fr
[1] R. Saito, G. Dresselhaus, and M. S. Dresselhaus, in Physical properties of carbon nanotubes, Imperial College Press (1998).

[2] M. Rohlfing and S.G. Louie, Phys. Rev. B 62, 4927 (2000).

[3] V. Perebeinos, J. Tersoff, and P. Avouris, Phys. Rev. Lett. 92, 257402 (2004).

[4] F. Wang et al., Science 308, 838 (2005).

[5] J. Maultzsch et al., Phys. Rev. B 72, 241402(R) (2005).

[6] M. Bayer, S. N. Walck, T. L. Reinecke, and A. Forchel, Phys. Rev. B 57, 6584 (1998).

[7] W. Braun, M. Bayer, A. Forchel, H. Zull, J. P. Reithmaier, A. I. Filin, and T. L. Reinecke, Phys. Rev. B 56, 12096 (1997).

[8] W. Langbein, H. Gislason, and J. M. Hvam, Phys. Rev. B 60, 16667 (1999)
[9] S. Rudin, T. L. Reinecke, and B. Segall, Phys. Rev. B 42, 11218 (1990).

[10] C. Piermarocchi, F. Tassone, V. Savona, A. Quattropani, and P. Schwendimann, Phys. Rev. B 53, 15834 (1996).

[11] V. Perebeinos, J. Tersoff, and P. Avouris, Phys. Rev. Lett. 94, 027402 (2005).

[12] S. Berciaud, C. Voisin, H. Yan, B. Chandra, R. Caldwell, Y. Shan, L. E. Brus, J. Hone, and T. F. Heinz, Phys. Rev. B 81, 041414(R) (2010).

[13] C. Galland, A. Högele, H. E. Tureci, and A. Imamoğlu, Phys. Rev. Lett. 101, 067402 (2008).

[14] A. Hartschuh, H. N. Pedrosa, L. Novotny, and T. D. Krauss, Science 301, 1354 (2003).

[15] M. Y. Sfeir, F. Wang, L. Huang, C.-C. Chuang, J. Hone, S. P. O'Brien, T. F. Heinz, and L. E. Brus, Science 306, 5701 (2004). 
[16] H. Htoon, M. J. O'Connell, P. J. Cox, S. K. Doorn, and V. I. Klimov, Phys. Rev. Lett. 93, 027401 (2004).

[17] K. Matsuda, T. Inoue, Y. Murakami, S. Maruyama, and Y. Kanemitsu, Phys. Rev. B 77, 033406 (2008).

[18] K. Yoshikawa, R. Matsunaga, K. Matsuda, and Y. Kanemitsu, Appl. Phys. Lett. 94, 093109 (2009).

[19] S. Berciaud, L. Cognet, and B. Lounis, Phys. Rev. Lett. 101, 077402-077405 (2008).

[20] Y.-Z. Ma, M. W. Graham, G. R. Fleming, A. A. Green, and M. C. Hersam, Phys. Rev. Lett. 101, 217402 (2008).

[21] O. Jost et al., Appl. Phys. Lett. 75, 2217 (1999).

[22] D. Mawhinney et al., Chem. Phys. Lett. 324, 213 (2000).

[23] J.S. Lauret et al., Phys. Rev. Lett. 90, 057404 (2003).

[24] M. O'Connell et al., Science 297, 593 (2002).

[25] J.S. Lauret et al., Physica E 21, 1057 (2004).

[26] S. Berger, C. Voisin, G. Cassabois, C. Delalande, Ph. Roussignol, and X. Marie, Nano Lett. 7, 398-402 (2007).

[27] D.C. Trung, W. Wuster, Ph. Roussignol, C. Voisin, G. Cassabois, M. Tchernycheva, F. Juline, F. Guillot, and E. Monroy, Appl. Phys. Lett. 97, 061903 (2010).
[28] S. Schmitt-Rink, D. S. Chemla, and D. A. B. Miller, Phys. Rev. B 32, 6601 (1985).

[29] D.C. Trung, C. Voisin, Ph. Roussignol, C. Roquelet, J. S. Lauret, and G. Cassabois, submitted for publication.

[30] W. Demtröder, Laser spectroscopy (Berlin SpringerVerlag, Third Edition, 2003).

[31] J. C. Meyer, M. Paillet, T. Michel, A. Morac, A. Neumann, G. S. Duesberg, S. Roth, and J.-L. Sauvajol, Phys. Rev. Lett. 95, 217401 (2005).

[32] H. Suzuura and T. Ando, Phys. Rev. B 65, 235412 (2002).

[33] G. Pennington and N. Goldsman, Phys. Rev. B 71, 205318 (2005).

[34] Y. Yin et al., Phys. Rev. Lett. 98, 037404 (2007).

[35] A. P. Shreve et al., Phys. Rev. Lett. 98, 037405 (2007).

[36] J. Jiang et al., Phys. Rev. B 72, 235408 (2005). 